VIRAL IMMUNITY

\section{This week's No. 1}

Competition for the number-one slot in the musical charts is fierce and the success of any song is affected by which other songs are released at the same time. Similarly, the course of HIV disease is influenced by infections that occur at the same time as HIV-1. Associations between human herpesvirus 6 (HHV-6) and HIV-1 have been reported, but whether these two viruses directly influence each other was unknown. In Nature Medicine, Grivel and colleagues now report that HHV-6 markedly affects the course of HIV infection, and they describe a potential mechanism for this effect.

HIV infects target cells using a receptor complex formed from CD4 and a chemokine receptor. Early stages of HIV-1 infection are commonly mediated by slow, macrophage-tropic variants that use the chemokine receptor CCR5, but at later stages the more pathogenic T-cell-tropic variants that use CXCR4 are detected (a phenomenon known as co-receptor switching).

To study the effect of HHV-6 on HIV-1 infection, the authors infected blocks of human tonsillar tissue with HHV-6 and one of two strains of HIV, a CXCR4-utilizing variant LAV.04, or a CCR5-utilizing variant SF162. They found that co-infection with HHV-6 suppressed replication of SF162, but had no effect, or even

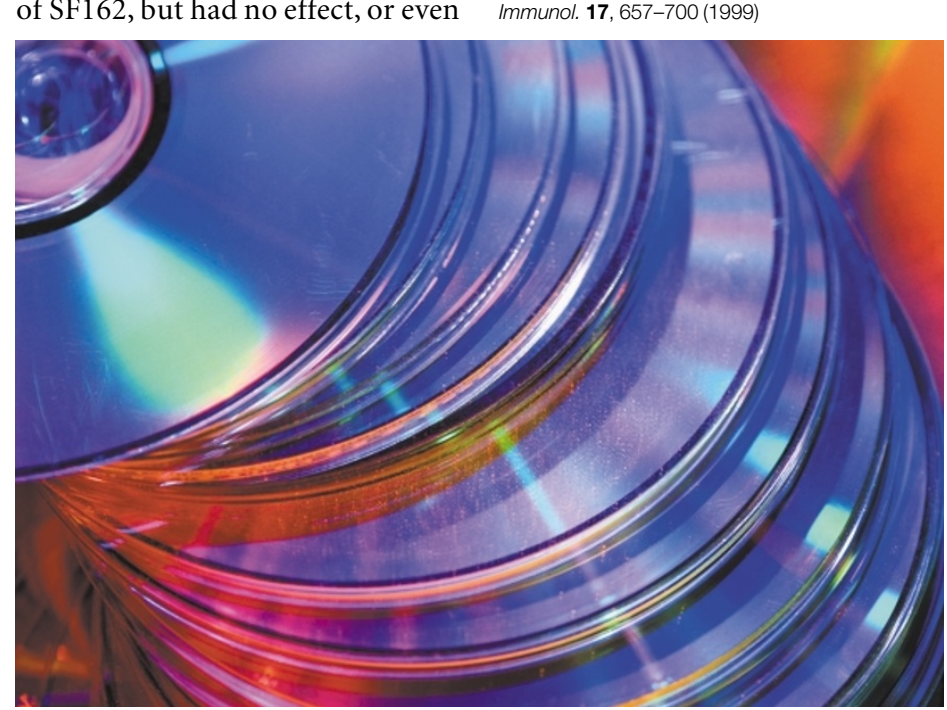

enhanced, replication of LAV.04. They also co-infected tissue blocks with HHV-6 and a mixture of LAV.04 and SF162. Again, replication of the CCR5utilizing SF162 was inhibited, but replication of the CXCR4-utilizing LAV.04 was unaffected.

But what is the mechanism of the effects of HHV-6 on HIV-1 infection? The authors investigated the production of CC chemokines that bind to CCR5 and have HIVinhibitory effects. HHV-6 infection alone, or with HIV-1, resulted in increased production of CCL5, the most potent HIV-inhibitory CC chemokine, whereas the levels of CCL3 and CCL4 did not change. This indicates that HHV-6 might selectively suppress the replication of CCR5-utilizing variants of HIV by upregulating CCL5 production.

So, infection with HHV-6, which frequently occurs in HIV-infected on the progression of HIV disease and these effects might be relevant to the HIV co-receptor switch that occurs at later stages of HIV disease. Jenny Buckland

\section{(2) References and links} ORIGINAL RESEARCH PAPER Grivel, J. et $a$ l. Suppression of CCR5- but not CXCR4-tropic HIV-1 in Iymphoid tissue by human herpesvirus 6 . Nature Medicine 7, 1232-1235 (2001)

FURTHER READING Berger, E. A. et al.

Chemokine receptors as HIV-1 coreceptors: roles in viral entry, tropism, and disease. Annu. Rev. Immunol. 17, 657-700 (1999) patients, might have profound effects

\section{N BRIEF}

\section{T-CELL DEVELOPMENT}

The critical role of LIGHT, a TNF family member, in T cell development.

Wang, J. et al. J. Immunol. 167, 5099-5105 (2001)

Negative selection and deletion of autoreactive thymocytes within the thymus are crucial for the maintenance of central tolerance. Here, the role of the tumour-necrosis factor family member LIGHT in these poorly defined processes is investigated. Through the generation of LIGHT transgenic mice, and by blocking the action of LIGHT in T-cell receptor transgenic mice, the authors establish that LIGHT has a crucial role in thymic negative selection.

\section{LYMPHOCYTE ACTIVATION}

Defective T cell activation and autoimmune disorder in Stra13-deficient mice.

Sun, H., Lu, B., Li, R. Q., Flavell, R. A. \& Taneja, R. Nature Immunol. 2, 1040-1047 (2001)

Taneja and colleagues describe the generation and analysis of mice deficient for the helix-loop-helix transcription factor, Stra13. At birth, and for the first few months of life, these mice were healthy, but with age a chronic lupus-like autoimmune disease developed. Several stages of CD4 T-cell activation and effector T-cell function were defective in these mice. In addition, activated $\mathrm{T}$ and $\mathrm{B}$ cells were not efficiently deleted, resulting in lymphoid organ hyperplasia and autoimmunity.

\section{PARASITE IMMUNOLOGY}

Modulation of blood fluke development in the liver by hepatic CD4+ lymphocytes.

Davies, S. J. et al. Science 294, 1358-1361 (2001)

For some helminths, it is known that host factors can trigger alternate developmental pathways that facilitate parasite survival in adverse conditions. The trematode parasite Schistosoma mansoni has such a pathway, which is triggered by host immune deficiency. Interestingly, the absence of a previously unidentified population of $\mathrm{CD}^{+}$hepatic $\mathrm{T}$ cells is the immune signal that triggers the alternative development pathway.

\section{IMMUNE REGULATION}

Membrane-bound TNF supports secondary lymphoid organ structure but is subservient to secreted TNF in driving autoimmune inflammation.

Ruuls, S.R. et al. Immunity 15, 533-543 (2001)

Tumour-necrosis factor (TNF) is a cytokine with pleiotrophic effects in the immune system. To distinguish between the in vivo roles of secreted and membrane-bound TNF, Sedgwick and coworkers created membrane-TNF 'knock-in' mice. Membrane TNF was found to be sufficient for normal development of secondary lymphoid organs, but the inflammatory disease experimental autoimmune encephalomyelitis was less severe in these mice, indicating that secreted TNF is important in inflammatory immune responses. 